\title{
Diagnostic yield of cerebrospinal fluid analysis in status epilepticus: an 8-year cohort study
}

\author{
Tolga D. Dittrich ${ }^{1}$ - Sira M. Baumann ${ }^{1}$ - Saskia Semmlack ${ }^{1}$ - Gian Marco De Marchis ${ }^{2,3,4}$ (1) - Sabina Hunziker ${ }^{1,3,5}$.

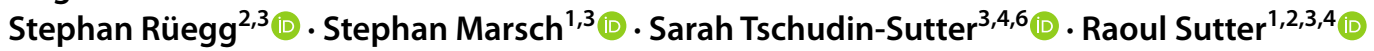

Received: 10 January 2021 / Revised: 3 February 2021 / Accepted: 4 February 2021 / Published online: 5 March 2021

(c) The Author(s) 2021

\begin{abstract}
Background We investigate the frequency and diagnostic yield of cerebrospinal fluid (CSF) analysis in adult patients with status epilepticus (SE) and its impact on the outcome.

Methods From 2011 to 2018, adult patients treated at the University Hospital Basel were included. Primary outcomes were defined as the frequency of lumbar puncture and results from chemical, cellular, and microbiologic CSF analyses. Secondary outcomes were differences between patients receiving and not receiving lumbar puncture in the context of SE.

Results In $18 \%$ of 408 patients, a lumbar puncture was performed. Of those, infectious pathogens were identified in $21 \%$ with $15 \%$ detected $\pm 24 \mathrm{~h}$ around SE diagnosis. $74 \%$ of CSF analyses revealed abnormal chemical or cellular components without infectious pathogens. Screening for autoimmune diseases was only performed in $22 \%$. In 8\%, no or late (i.e., $>24$ after SE diagnosis) lumbar puncture was performed despite persistent unknown SE etiology in all, transformation into refractory SE in $78 \%$, and no recovery to premorbid neurologic function in $66 \%$. Withholding lumbar puncture was associated with no return to premorbid neurologic function during hospital stay independent of potential confounders. Not receiving a lumbar puncture was associated with presumed known etiology and signs of systemic infectious complications.

Conclusions Withholding lumbar puncture in SE patients is associated with increased odds for no return to premorbid neurologic function, and CSF analyses in SE detect infectious pathogens frequently. These results and pathologic chemical and cellular CSF findings in the absence of infections call for rigorous screening to confirm or exclude infectious or autoimmune encephalitis in this context which should not be withheld.
\end{abstract}

Keywords Status epilepticus $\cdot$ Intensive care $\cdot$ Neurocritical care $\cdot$ Lumbar puncture $\cdot$ Cerebrospinal fluid

Raoul Sutter

raoul.sutter@usb.ch

Tolga D. Dittrich

tolga.dittrich@usb.ch

Sira M. Baumann

siramaria.baumann@usb.ch

Saskia Semmlack

saskia.semmlack@usb.ch

Gian Marco De Marchis

gian.demarchis@usb.ch

Sabina Hunziker

sabina.hunziker@usb.ch

Stephan Rüegg

stephan.rueegg@usb.ch

Stephan Marsch

stephan.marsch@usb.ch
Sarah Tschudin-Sutter

sarah.tschudin@usb.ch

1 Department of Intensive Care Medicine, University Hospital Basel, Basel, Switzerland

2 Department of Neurology, University Hospital Basel, Basel, Switzerland

3 Medical Faculty of the University of Basel, Basel, Switzerland

4 Department of Clinical Research, University of Basel, Basel, Switzerland

5 Department of Medical Communication and Psychosomatic Medicine, University Hospital Basel, Basel, Switzerland

6 Division of Infection Diseases and Hospital Epidemiology, University Hospital Basel, Basel, Switzerland 


\section{Background}

Status epilepticus (SE) is a neurologic emergency with high morbidity and mortality [23, 25, 27]. Since the etiology has a significant if not the most important effect on survival $[23,26]$ and identification of the underlying cause guides treatment, rapid and reliable diagnostic measures and workup are essential. While important investigations by means of anamnesis, physical and laboratory examination, as well as neuroimaging may uncover underlying SE etiologies, patients with unremarkable exams represent great challenges for the treating team. In these situations, every effort must be made to identify the cause of SE.

Encephalitis represents an important SE etiology that may come with normal or discrete and unremarkable neuroimaging and otherwise rather non-specific anamnesis, physical status, and laboratory findings. Although the detection of infectious pathogens in the cerebrospinal fluid (CSF) is the "gold standard" for the diagnosis of infectious (meningo-) encephalitis [6], CSF analyses are not consistently recommended in international guidelines regarding SE. While the Neurocritical Care Society Status Epilepticus Committee suggests considering the performance of a lumbar puncture in such clinical scenarios [4], CSF analyses are neglected by the guidelines of the European Federation of Neurological Societies [15] and the most recent evidence-based guideline on the treatment of convulsive SE in children and adults of the American Epilepsy Society [11]. As infectious (meningo-)encephalitis encompasses many different viral and bacterial infections of the central nervous system (CNS) [6] and accounts for up to 10\% of SE etiologies (not accounting for missed or underdiagnosed cases) [28, 35], the question arises whether or not CSF analyses should be labeled as mandatory with persistent unknown etiology, as already recommended for the diagnosis of new-onset refractory SE (NORSE) or cryptogenic NORSE [13, 34]. This comes with uncertainty once CSF is analysed regarding the extent to which chemical and cellular changes may be caused by SE per se and not by underlying infectious or autoimmune diseases. Despite these worrisome facts and uncertainties, studies in this context are lacking.

We aimed to investigate the frequency and diagnostic yield of cerebrospinal fluid analysis in adult patients with SE.

\section{Materials and methods}

This observational study was performed at the University Hospital of Basel, a Swiss tertiary academic medical care center. The STROBE-guidelines were followed to enhance the quality and standardization for the reporting of observational studies [32]. The Swiss ethics committee (Ethikkommission Norwest- und Zentralschweiz) approved the study in compliance with the ethical standards laid down in the 1964 Declaration of Helsinki and its later amendments and waived patients' consent.

\section{Data collection}

Data analysed in this study are part of the ongoing STatus EPilepticus Unicenter Population (STEP UP) study (Clinicaltrials.gov No. NCT04348318). From January 1st, 2011 to December 31st, 2018, clinical, laboratory, and epileptologic data of all consecutive adult SE patients ( $\geq 18$ years of age) were assessed with the digital institutional electroencephalographic (EEG) and medical databases. Patients with SE in the context of acute hypoxic-ischemic encephalopathy were excluded.

The following clinical data were collected: age, sex, etiology (categorized as potential non-fatal and fatal as defined elsewhere [17]), quantified comorbidities by the Charlson Comorbidity Index [5], intubation and duration of mechanical ventilation, the use of antiseizure drugs and continuously administered anesthetics, and complications emerging during SE were noted. In addition, the time of performance of a lumbar puncture and results from chemical and microbiologic analyses of the cerebrospinal fluid were assessed. Severity of SE was quantified by the most frequently validated Status Epilepticus Severity Score (STESS) as previously described $[18,19,22,24]$. Performance and types of neuroimaging [i.e., cerebral magnetic resonance imaging (MRI) and/or computed tomography (CT)] were assessed. Pathologic findings, as described after the images have been examined by two radiologists were noted. Finally, the length of the intensive care unit (ICU) and hospital stay were recorded.

\section{Classification of SE and SE duration}

Classification of SE followed the guidelines of the task force on the classification of SE of the International League Against Epilepsy [29]. SE types were categorized as focal nonconvulsive SE without coma with or without altered consciousness and absence SE, SE with motor symptoms (including myoclonic and convulsive forms), and nonconvulsive SE with coma.

SE duration was defined as the time span between the clinical and/or EEG evidence of seizure onset and the timepoint at which seizure termination was proven by EEG. SE duration is expressed as a $12 \mathrm{~h}$ approximation, as two different EEG recording strategies were used, such as continuous EEG or spot EEGs of $\geq 30$ min every $12 \mathrm{~h}$. Continuous EEG was consistently used for refractory SE. 


\section{Detection of infectious pathogens, chemical and cellular CSF analyses}

The microbiologic workup and the diagnoses of (meningo-) encephalitis were systematically established according to the pre-established frameworks [6, 7]. The diagnostic algorithms for infectious (meningo-)encephalitis of the consensus statement of the international encephalitis consortium were followed [30] and based on the detection of viral ribonucleic acid (RNA) or deoxyribonucleic acid (DNA) of infectious pathogens with PCR in the CSF. The diagnosis of bacterial (meningo-)encephalitis was established with the microscopic detection of Gram-stained infectious pathogens in the CSF, the detection of aerobic and anaerobic bacterial cultures for 6 days, and/or PCR. Due to local conditions, two additional diagnostic procedures were applied. First, the diagnosis of Borrelia burgdorferi infection was established according to the national guidelines by the detection of intrathecal antibodies [8]; second, the diagnosis of tickborne encephalitis ("Frühsommer" Meningoencephalitis, FSME) was diagnosed with positive serology [31].

Chemical analyses, such as the CSF concentrations of proteins, glucose (including the glucose ratio CSF/serum), lactate, IgG, IgA, and IgM Indexes and quotients and Reiber diagrams, oligoclonal bands, and screening for autoantibodies (including antibodies against $N$-methyl-D-aspartate [NMDA]-receptor, voltage-gated-kalium-channels [VGKC], and glutamic acid decarboxylase (GAD)) were assessed. In addition, information from screening for paraneoplastic antibodies including anti-Hu (ANNA-1)-, anti-Yo (PCA1)-, anti-Ri (ANNA-2)-, anti-CV2 (CRMP5)-, anti-Ma1 (PNMA1)-, anti-Ma2/Ta (PNMA2)-, and anti-amphiphysinantibodies were collected. Screening for autoantibodies and paraneoplastic antibodies was not restricted to the CSF and was also performed serum samples.

During the study period, all CSF were analyzed regarding cellular and chemical characteristics including levels of lactate, glucose, proteins, Gram staining, and bacterial cultures. Further analyses regarding immunoglobulin synthesis, PCR for detection of infectious pathogens, screening for autoantibodies were not standardized, as during the study period, no institutional algorithms were in place. Hence, further analyses, including the performance of IgG, IgA or IgM quotient diagrams and Reiber diagrams, PCRs, and screening for autoantibodies were performed at the discretion of the treating and/or consulting physicians.

\section{Outcomes}

Primary outcomes were defined as the frequency of lumbar puncture and results from chemical and microbiologic cerebrospinal fluid analyses.
Differences between patients receiving and not receiving lumbar punctures and associations between lumbar puncture and return to premorbid neurologic function during hospital stay were considered secondary outcomes.

\section{Statistics}

Patients were categorized into patients with and without lumbar puncture during their hospital stay. Univariable comparison of patients with and without lumbar puncture was performed by the Chi-square test or the Fisher's exact test. For continuous variables, the Shapiro-Wilk test was used to distinguish between normally and not normally distributed variables. Normally distributed variables were analyzed with the Student's $t$ test, whereas variables violating the normal distribution were analyzed with the Mann-Whitney $U$ test.

Univariable logistic regression models were applied to calculate odds ratios for the associations of not having received a lumbar puncture with no return to premorbid neurologic function. Multivariable logistic regression model was subsequently performed to adjust the association between no performance of lumbar puncture and no return to premorbid neurologic function for potential confounders including potential fatal etiology, STESS, duration of SE, the use of continuous anesthetics, and systemic infectious complications (as detected in the univariable comparison between patients receiving and not receiving lumbar punctures).

Two-sided $p$-values $\leq 0.05$ were considered significant. Statistical analysis was performed with STATA ${ }^{\circledR} 16.1$ (Stata Corp., College Station, TX, USA).

\section{Data availability}

Anonymized data are available from the corresponding author and will be shared on reasonable request from any qualified investigator.

\section{Results}

From January 2011 to December 2018, 469 adult patients were treated for SE in the University Hospital Basel. Of those, 61 (13\%) developed SE from hypoxic-ischemic brain injury following cardiorespiratory arrest and were excluded from the study (Fig. 1).

\section{Primary outcomes}

Of the remaining 408 adult patients with SE, lumbar puncture was performed during their hospital stay in 72 patients (17.6\%) within a median of 0 days [interquartile range (IQR) 0-1] from SE diagnosis. Of those, infectious pathogens were identified in 15 patients $(20.9 \%)$, with 
Fig. 1 Flow chart and performance of lumbar puncture in patients with status epilepticus. $L P$ lumbar puncture
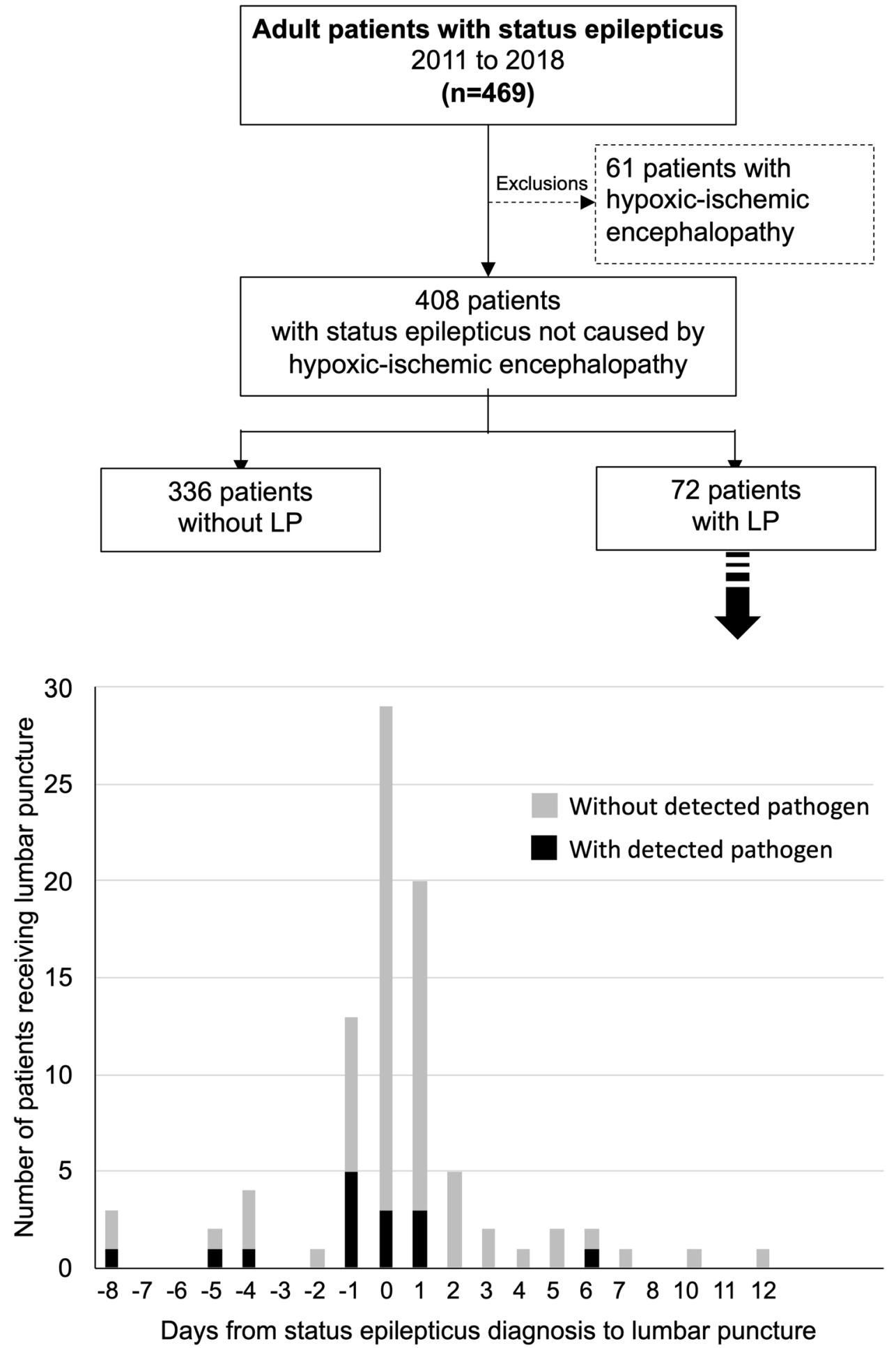

11 being detected $\pm 24 \mathrm{~h}$ around SE diagnosis $(73.3 \%$ of detected infections). Detailed information regarding the detected infectious pathogens and pathologic chemical and cellular components of the CSF in patients without infectious pathogens or identified autoimmune disease are presented in Fig. 2. Of all 72 CSF analyzed, 53 (74\%) showed abnormal increases of either chemical (i.e., protein and/or lactate concentrations) or cellular (i.e., mono- and/ or polynuclear leukocytes) components but no infectious pathogens and no evidence of autoimmune disease involving the nervous system. In patients receiving lumbar puncture, neuroimaging was performed in 57 (79\%), including $43(60 \%)$ MRI and 57 (79\%) CT. Of those, signs of possible cerebral inflammation were found in $3(5 \%)$, signs of 
Fig. 2 Diagnostic yield of cerebrospinal fluid analyses in patients without infectious pathogens or autoimmune encephalitis. CSF cerebrospinal fluid
Diagnostic yield of cerebrospinal fluid analyses

in patients with status epilepticus

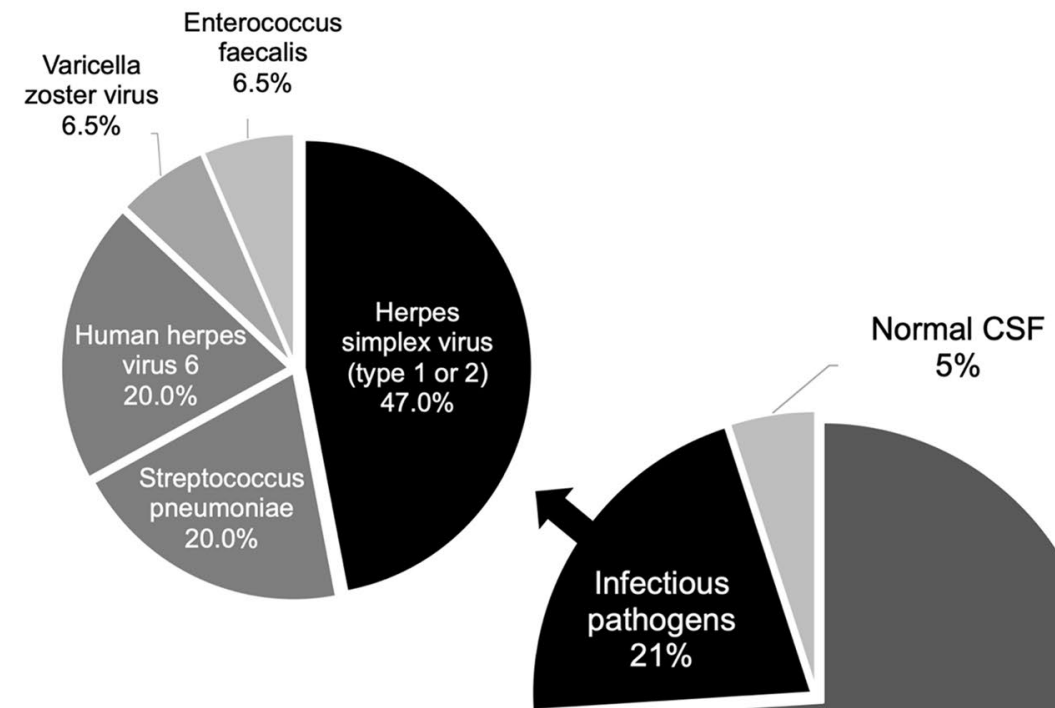

Pathologic CSF without infectious

pathogen or

autoimmune disease $74 \%$

Heat map regarding pathologic CSF findings in patients with status epilepticus without pathogens or autoimmune encephalitis

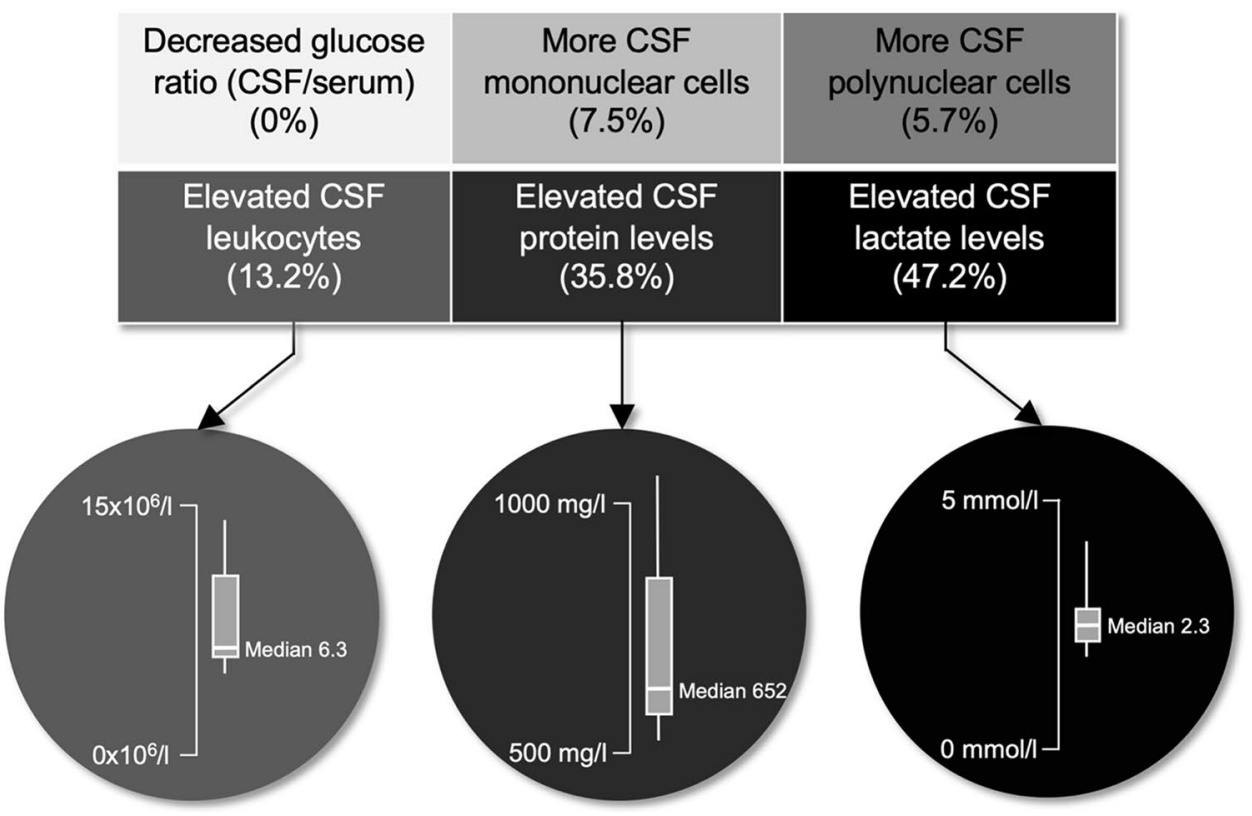


acute ischemic strokes in $2(3 \%)$, and signs of brain tumors in $4(7 \%)$ patients.

Figure 3 presents further details regarding the performance of timely (i.e., within $24 \mathrm{~h}$ after SE diagnosis) and late (i.e., after $24 \mathrm{~h}$ after SE diagnosis) lumbar puncture in patients with different constellations regarding known or unknown etiologies. Infectious pathogens were detected in $21 \%$ of patients with CSF analyses, with most pathogens being herpes simplex virus type 1 or 2 , followed by streptococcus pneumoniae.

Remarkably, in 32 patients (7.9\%) no or late lumbar puncture was performed despite having a persistent unknown etiology of SE in $100 \%$ and not having complete recovery to their premorbid neurologic function in $65.6 \%$ during their hospital stay. Of those 32 patients, 25 (78.2\%) SE was refractory with a duration of more than $24 \mathrm{~h}$, and nine patients developed cryptogenic NOSRE. Of those, lumbar puncture was performed in

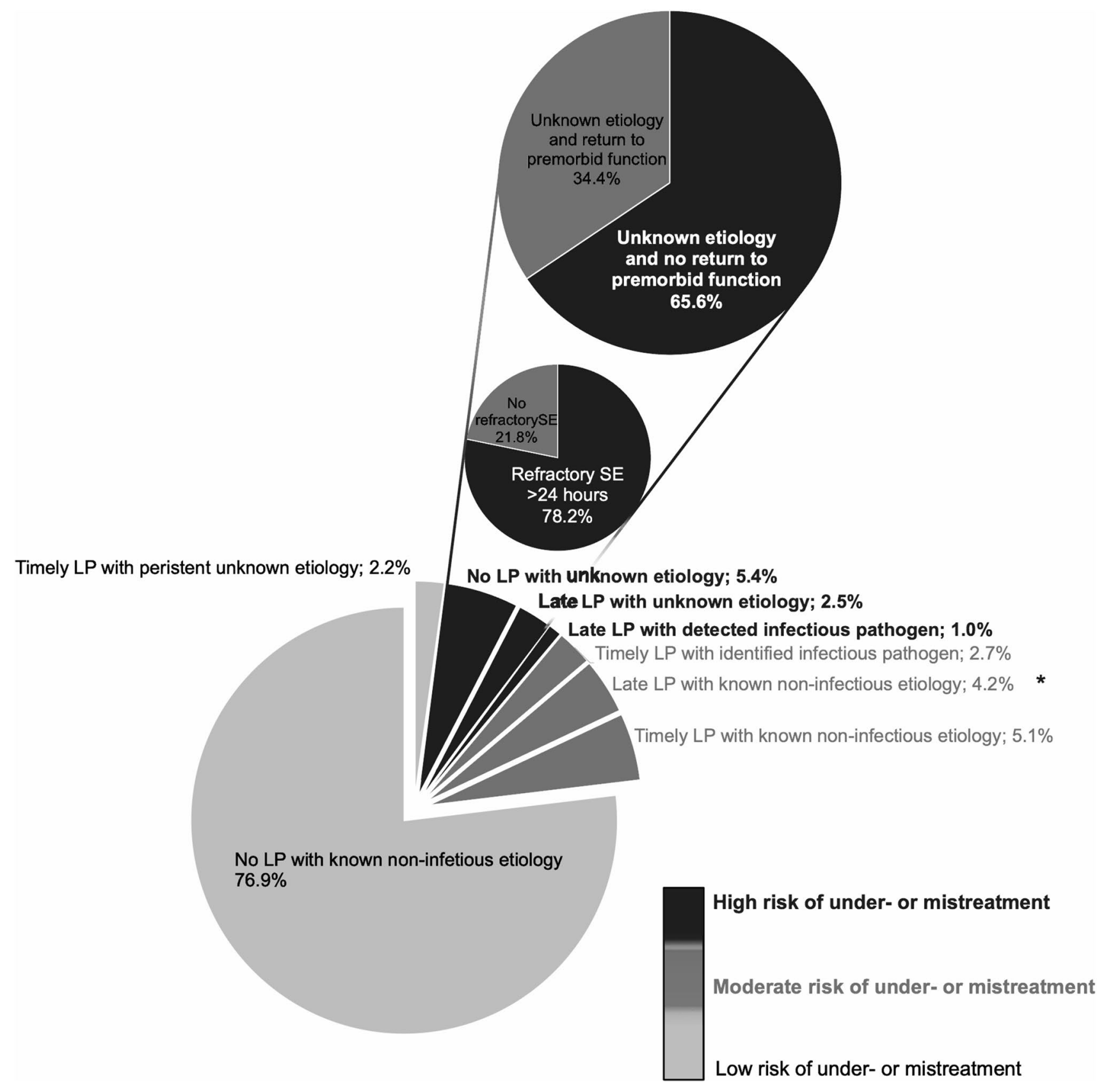

Fig. 3 Performance of lumbar puncture in patients with status epilepticus. $L P$ lumbar puncture, late $L P$ lumbar puncture after $24 \mathrm{~h}$ following the diagnosis of SE. *Of patients with late LP with identified non-infectious etiology, one had NMDA encephalitides. In all others, SE etiology was known prior to LP 
only three (30\% of NOSRE) without detection of infectious pathogens, no oligoclonal bands or an increased IgG index, and only two (22\% of NOSRE) patients were screened for anti- $N$-methyl-D-aspartate (NMDA)-receptor antibodies in the CSF and serum revealing negative results. In our total cohort, two patients were diagnosed with having developed SE from anti-NMDA-receptor antibody encephalitis in whom the antibodies were detected in their serum. Screening for oligoclonal bands and IgG index, IgG and Reiber diagrams were performed in 16 (22\% of patients with lumbar puncture) patients with all having no elevated oligoclonal bands and none having elevated IgG. Antibody screening of the CSF and serum was also only performed in nine patients (13\% of patients with lumbar puncture) regarding anti-NMDA-receptor antibodies, including additional screening for anti-voltagegated-kalium-channels (VGKC)-antibodies in 8 and one for anti-glutamic acid decarboxylase (GAD) antibodies. Screening for paraneoplastic antineuronal antibodies in the serum was performed in 5 patients, including anti-Hu (ANNA-1)-, anti-Yo (PCA-1)-, anti-Ri (ANNA-2)-, anti-CV2 (CRMP5)-, anti-Ma1 (PNMA1)-, anti-Ma2/Ta (PNMA2)-, and anti-amphiphysinantibodies. Detailed information regarding the diagnostic CSFworkup is presented in the online supplemental Table 1.

\section{Secondary outcomes}

Comparison of clinical characteristics, treatment, course, and outcome of patients with and without lumbar puncture are presented in Table 1. In patients with lumbar puncture, the etiology of SE was unknown more frequently, SE was less frequently convulsive, duration of SE and ICU treatment was longer, and infections were detected more often. Uni- and multivariable analyses regarding the association between no performance of lumbar puncture and outcome are presented in Table 2 (upper part). Univariable analysis revealed no significant association between withholding lumbar puncture and no return to premorbid neurologic function. When adjusting for potential confounders as detected in the comparison between patients with and without lumbar puncture (as shown in Table 1), withholding lumbar puncture was found to be an independent association with no return to premorbid neurologic function.

Further uni- and multivariable analyses revealed that known etiology and absence of infectious complications were independent associations with patients not receiving a lumbar puncture (Table 2, lower part).

\section{Discussion}

Despite current recommendations regarding the management of status epilepticus [4], lumbar puncture was inconsistently performed in our patients in whom the other diagnostic steps have not clarified the etiology of SE and whose neurological condition has not fully recovered to premorbid status. Our study further revealed that infectious pathogens were identified frequently once CSF analysis was performed. The most commonly detected pathogens were herpes simplex virus type 1 or 2 , streptococcus pneumoniae, and human herpes virus 6 . While the latter seems surprising in adult patients, the fact that our institution treats a large number of immunosuppressed and hematologic patients is a possible explanation, as they are susceptible to infections with or reactivation of less common pathogens such as human herpes virus 6 [33]. As most detected infectious encephalitides are potentially treatable, the underperformance of CSF analyses seems critical and may also be at least a partial explanation of why withholding a lumbar puncture was independently associated with increased odds for no return to premorbid neurologic function. Our results further indicate that presumed known etiology and the absence of infectious complications were independently associated with increased odds of not receiving a lumbar puncture. This indicates that clinicians are more likely to withhold lumbar puncture with the absence of clinical signs of infections, a critical decision especially in the context of viral or autoimmune encephalitis, which often emerges without clinical signs of inflammation. Although the association between not performing a lumbar puncture and outcome seems plausible at first glance, our results have to be interpreted with caution, as our analyses cannot exclude clinical scenarios in which clinicians had a supportive rationale for withholding diagnostic procedures, such as when the likelihood of an expected poor outcome is presumed to be high. Another scenario in which lumbar puncture is not performed with good reason is the presence of a cerebral mass with signs of increased intracranial pressure or brainstem compression on neuroimaging - a situation not described in the neuroimaging in any of our patients, not even in the patients with brain tumors and neuroimaging.

The underuse of CSF analyses may also be explained by the fact that during the study period no institutional guidelines regarding the performance of lumbar punctures were in place and that the guidelines of the Neurocritical Care Society only provide vague recommendations that lumbar puncture should be considered in specific cases, and most other SE guidelines do not even mention the lumbar puncture and CSF analyses as an important diagnostic step [11, 15]. Our finding that infectious pathogens were identified in every fifth patient in whom CSF was analyzed indicates that in SE patients without known etiology, infections of the CNS are frequent and may be underestimated due to the underuse of CSF analyses. This comes along with a high probability of underdiagnosed autoimmune diseases as screening for autoimmune diseases was not performed systematically in our patients with persistent unknown etiology of SE. Although it is unclear why current guidelines "toned down" or neglected 
Table 1 Characteristics of patients with status epilepticus with and without lumbar puncture $(n=408)$

\begin{tabular}{|c|c|c|c|c|c|c|c|}
\hline $\begin{array}{l}\text { Characteristics } \\
\text { Demographics and admission characteristics }\end{array}$ & \multicolumn{2}{|c|}{$\begin{array}{l}\text { Total cohort } \\
(n=408)\end{array}$} & \multicolumn{2}{|c|}{$\begin{array}{l}\text { Patients without } \\
\text { lumbar puncture } \\
(n=336)\end{array}$} & \multicolumn{2}{|c|}{$\begin{array}{l}\text { Patients with lumbar } \\
\text { puncture }(n=72)\end{array}$} & $p$-value \\
\hline Age (years; median, IQR) & 67.2 & $54-78$ & 68.2 & $54-78$ & 65.6 & $54-74$ & 0.325 \\
\hline Sex (female; $n, \%)$ & 198 & 48.5 & 160 & 47.6 & 38 & 52.8 & 0.427 \\
\hline \multicolumn{8}{|l|}{ Clinical features } \\
\hline \multicolumn{8}{|l|}{ SE etiology $(n, \%)$} \\
\hline Known etiology & 367 & 89.9 & 314 & 93.5 & 53 & 73.6 & $<0.001$ \\
\hline Confirmed potential fatal etiology (not mutually exclusive) & 134 & 32.8 & 109 & 32.4 & 25 & 34.7 & 0.708 \\
\hline Fast growing brain tumors & 34 & 8.3 & 30 & 8.9 & 4 & 5.6 & \\
\hline Acute intracranial hemorrhage & 41 & 10.0 & 39 & 11.6 & 2 & 2.8 & \\
\hline Infectious (meningo-)encephalitis & 15 & 20.8 & 0 & 0.0 & 15 & 20.8 & \\
\hline Acute ischemic stroke & 16 & 3.9 & 14 & 4.2 & 2 & 2.8 & \\
\hline Acute severe traumatic brain injury & 12 & 2.9 & 12 & 3.6 & 0 & 0.0 & \\
\hline Acute autoimmune encephalitis & 2 & 0.5 & 1 & 0.3 & 1 & 1.4 & \\
\hline Septic encephalitis & 5 & 1.2 & 4 & 1.2 & 1 & 1.4 & \\
\hline Others & 9 & 2.2 & 7 & 2.1 & 2 & 2.8 & \\
\hline Unknown etiology & 41 & 10.0 & 22 & 6.5 & 19 & 26.4 & $<0.001$ \\
\hline \multicolumn{8}{|l|}{ SE type\# $(n, \%)$} \\
\hline Focal NCSE without coma & 167 & 40.9 & 140 & 41.7 & 27 & 37.5 & 0.514 \\
\hline With altered consciousness & 145 & 35.5 & 123 & 36.6 & 22 & 30.6 & \\
\hline Without altered consciousness & 22 & 5.4 & 17 & 5.1 & 5 & 6.9 & \\
\hline SE with motor symptoms (convulsive, myoclonic) & 129 & 31.6 & 114 & 33.9 & 15 & 20.8 & 0.034 \\
\hline Convulsive SE & 87 & 21.3 & 77 & 22.9 & 10 & 13.9 & \\
\hline Myoclonic SE & 42 & 10.3 & 37 & 11.0 & 5 & 6.9 & \\
\hline NCSE with coma & 112 & 27.5 & 82 & 24.4 & 30 & 41.7 & 0.003 \\
\hline NCSE with coma (non-subtle) & 80 & 19.6 & 60 & 17.9 & 20 & 27.8 & \\
\hline Subtle SE & 32 & 7.8 & 22 & 6.6 & 10 & 13.9 & \\
\hline Charlson Comorbidity Index* (median, IQR) & 2 & $1-4$ & 2 & $1-5$ & 2 & $1-4$ & 0.231 \\
\hline STESS (median, IQR)** & 3 & $2-4$ & 3 & $1-4$ & 3 & $2-4$ & 0.052 \\
\hline SE duration (days; median, IQR) & 1 & $0.5-2$ & 1 & $0.5-1$ & 1.5 & $0.75-3$ & $<0.001$ \\
\hline \multicolumn{8}{|l|}{ Treatment characteristics during SE } \\
\hline In-hospital treatment (days; median, IQR) & 13 & $6-22$ & 12 & $5-21.5$ & 14.5 & $8.5-24.5$ & 0.051 \\
\hline ICU treatment (days; median, IQR) & 2 & $1-6$ & 2 & $0-5$ & 4 & $2-10$ & 0.002 \\
\hline Patients with benzodiazepines $(n, \%)$ & 403 & 98.8 & 333 & 99.1 & 70 & 97.2 & \\
\hline Patients with second-line antiseizure drugs $(n, \%)$ & 396 & 97.1 & 324 & 96.4 & 72 & 100.0 & \\
\hline Number of non-anesthetic antiseizure drugs (median, IQR) & 3 & $2-4$ & 3 & $2-4$ & 3 & $2-4$ & 0.319 \\
\hline Mechanical ventilation $(n, \%)$ & 145 & 35.5 & 117 & 34.8 & 28 & 38.9 & 0.513 \\
\hline Duration of mechanical ventilation (days; median, IQR) & 5 & $2-13$ & 5 & $2-11$ & 9 & $4-14$ & 0.216 \\
\hline \multicolumn{8}{|l|}{ Complications during SE $(n, \%)$} \\
\hline Infections & 75 & 18.4 & 54 & 16.1 & 21 & 29.2 & 0.009 \\
\hline Arterial hypotension requiring vasopressors & 44 & 10.8 & 34 & 10.1 & 10 & 13.9 & 0.349 \\
\hline Cardiorespiratory resuscitation & 4 & 1.0 & 3 & 0.9 & 1 & 1.4 & \\
\hline Multiorgan failure & 3 & 0.7 & 3 & 0.9 & 0 & 0.0 & \\
\hline \multicolumn{8}{|l|}{ Outcomes $(n, \%)$} \\
\hline No return to premorbid neurologic function at discharge (incl. death) & 237 & 58.1 & 198 & 58.9 & 39 & 54.2 & 0.457 \\
\hline Death at hospital discharge & 30 & 7.4 & 25 & 7.4 & 5 & 6.9 & 1.000 \\
\hline
\end{tabular}

$C N S$ central nervous system, $S E$ status epilepticus, NCSE nonconvulsive status epilepticus, $C S E$ convulsive status epilepticus, ICU intensive care unit, $I Q R$ interquartile range

*Charlson Comorbidity Index [5]

**STESS $=$ Status Epilepticus Severity Score (Range 0-6) [18, 19, 22]

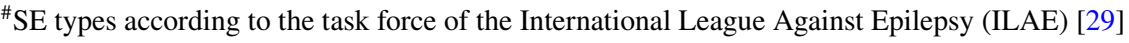


Table 2 Uni- and multivariable analyses regarding the associations with no return to premorbid neurologic function and no performance of lumbar puncture

\begin{tabular}{|c|c|c|c|c|c|c|}
\hline & \multicolumn{3}{|c|}{ Univariable analyses } & \multicolumn{3}{|c|}{ Multivariable analyses* } \\
\hline & OR & $95 \% \mathrm{CI}$ & $p$-value & OR & $95 \% \mathrm{CI}$ & $p$-value \\
\hline \multicolumn{7}{|c|}{ No return to premorbid neurologic function } \\
\hline No performance of lumbar puncture & 1.21 & $0.73-2.03$ & 0.458 & 2.25 & $1.18-4.27$ & 0.013 \\
\hline Potential fatal etiology & 2.51 & $1.61-3.92$ & $<0.001$ & 1.83 & $1.09-3.07$ & 0.023 \\
\hline Unknown etiology & 1.14 & $0.59-2.21$ & 0.693 & 1.16 & $0.51-2.66$ & 0.725 \\
\hline STESS & 1.76 & $1.52-2.04$ & $<0.001$ & 1.79 & $1.52-2.11$ & $<0.001$ \\
\hline Use of continuous anesthetics & 2.77 & $1.69-4.52$ & $<0.001$ & 2.36 & $1.33-4.19$ & 0.003 \\
\hline Duration of SE (per day) & 1.25 & $1.10-1.43$ & 0.002 & 1.22 & $1.08-1.39$ & 0.002 \\
\hline Systemic infectious complications & 1.95 & $1.13-3.35$ & 0.016 & 0.97 & $0.51-1.86$ & 0.928 \\
\hline \multicolumn{7}{|l|}{ No performance of lumbar puncture } \\
\hline Known etiology\# & 5.12 & $2.59-10.09$ & $<0.001$ & 5.79 & $2.79-12.01$ & $<0.001$ \\
\hline SE with motor symptoms\# & 1.95 & $1.06-3.60$ & $\mathbf{0 . 0 3 2}$ & 1.69 & $0.87-3.26$ & 0.120 \\
\hline STESS\# & 0.85 & $0.73-0.99$ & 0.042 & 0.92 & $0.78-1.08$ & 0.302 \\
\hline Use of continuous anesthetics\# & 0.49 & $0.29-0.84$ & 0.009 & 0.60 & $0.33-1.10$ & 0.101 \\
\hline Duration of SE (per day)\# & 0.98 & $0.93-1.02$ & 0.325 & 1.03 & $0.97-1.09$ & 0.363 \\
\hline Systemic infectious complications\# & 0.46 & $0.26-0.84$ & 0.010 & 0.47 & $0.24-0.91$ & 0.026 \\
\hline
\end{tabular}

SE status epilepticus, STESS Status Epilepticus Severity Score (Range 0-6) [18, 19, 22], OR odds ratio, CI confidence interval

\#All variables that significantly differed in the comparison of Table 1

*Hosmer-Lemeshow goodness of fit test $\mathrm{Chi}^{2} 13.43 ; p=0.100$ indicating adequate model fit

**Hosmer-Lemeshow goodness of fit test $\mathrm{Chi}^{2} 6.33 ; p=0.611$ indicating adequate model fit the importance of CSF analyses, our results suggest that CSF analyses should be performed more frequently, since many patients have a persistent undetermined etiology of SE and lumbar puncture is safe if neuroimaging reveals no signs of increased intracranial pressure.

The fact that lumbar puncture was less frequently performed, especially in patients with convulsive SE, may be explained by the fact that during convulsions invasive diagnostic procedures put the patients at additional risks of injury in conjunction with unpredictable motor activity of the patient. However, as most patients were in refractory SE for more than $24 \mathrm{~h}$ and treated with the induction of deep coma, this assumption may only be a partial explanation.

Another important finding is the large proportion of abnormal chemical and/or cellular CSF components in patients in whom microbiologic workup did not detect infectious pathogens. To what degree these CSF findings were explained by the inflammation as a result of an excessive intracerebral increase of neurotransmitters to neurotoxic concentrations due to SE per se or by undetected or unsuspected autoimmune CNS disease remains unclear, as screening for autoantibodies was left to the discretion of the clinician and was not performed systematically. Although glucose $\mathrm{CSF} /$ serum ratios and lactate concentrations were mostly unremarkable, CSF protein levels and leukocyte counts were frequently and noticeably increased even without detectable infectious pathogens. In this context, it is important to mention that according to a recent systematic review there is concordance in the available literature to recommend increasing CSF total protein upper reference limits and to consider implementing age-adjusted values above $600 \mathrm{mg} / \mathrm{L}$ starting at age 50 [3]. However, when talking this into account, only 6 patients with a protein level above $500 \mathrm{mg} / \mathrm{L}$ without proof of infectious or autoimmune encephalitis were then still considered within the normal range.

During the last decade, CSF examination for non-infectious etiologies of SE has expanded, uncovering a number of autoimmune diseases that can be linked to the emergence of autoimmune encephalitis driven SE, and therapeutics in instances have been tailored for specific findings. Remarkably, only a very small number of patients in our cohort were screened for autoantibodies in the CSF, and diagnosis of anti-NMDA-receptor encephalitis was diagnosed in only two of our patients. Although information regarding the screening of antibodies in the patients' blood samples could not be assessed, as these analyses were performed in out-of-hospital test centers during the study period, underestimation and missed diagnosis is obvious, especially for patients with severe conditions including NOSRE in whom investigations regarding non-infectious CNS disorders are crucial, lumbar puncture was only performed in every third patient. This is worrisome, as brain MRI studies can be unremarkable in more than half of patients with autoimmune encephalitides $[12,20]$ and autoimmune encephalitis is be detected in the context of NOSRE in up to $20 \%$ in prior studies [10]. To 
reduce the risk of missing or underdiagnosing infectious or autoimmune encephalitis in patients with SE, the authors propose an algorithm for the decision of whether to perform a lumbar puncture or not in patients with SE (Online supplemental Fig. 1). If the etiology of SE remains unclear after routine chemical, cellular and microbiologic analyses of the CSF, antibody screening should be performed to uncover autoimmune encephalitis as recently outlined [12].

\section{Limitations}

The single-center observational design limits the generalizability of this study. The fact, however, that the clinical characteristics in our population are similar to those in other SE studies, including age $[1,2,9,16,21]$, outcome [9, 14], etiologies [2, 9, 14, 21], SE severity [2, 9], and types of SE $[1,14,16]$ indicate that our cohort has several characteristics typical of other large SE cohorts.

Autoimmune diseases were not routinely screened in our institution, and most investigations are initiated upon suspicious patient's history and/or neuroimaging. Therefore, it remains unclear to what extent autoimmune diseases may have been missed and have at least partially explained the pathologic CSF findings. As our understanding regarding autoimmune encephalitis as a cause of SE has expanded in recent years, underestimation and missed diagnosis in this context is very likely, especially in the first years of our study. In addition, signs of activation of the immune system, such as the presence of plasmacells, large activated lymphocytes, or macrophages were not systematically documented in our institution. Another limitation comes from the fact that our study design does not allow any analyses regarding the number of patients receiving antimicrobials for suspected or proven CNS infection, as several patients were also treated for infectious complications outside the CNS and the differentiation in retrospect between antimicrobials only administered for one or the other is error prone and not possible in every patient. For the same reason, unfortunately, analyses regarding the effects of antimicrobial treatment on course and outcome would be subject to substantial bias.

Finally, the approximation of SE duration represents another limitation regarding potential underestimation of SE duration, especially with unwitnessed onset, mainly the case with nonconvulsive SE [27].

\section{Conclusions}

Withholding lumbar puncture in SE patients is associated with increased odds for no return to premorbid neurologic function, and CSF analyses in SE patients detect infectious pathogens frequently. These findings and the fact that pathologic CSF findings on a chemical and/or cellular level in the absence of systemic infections call for rigorous screening to confirm or exclude infectious or autoimmune encephalitis and should not be withheld. More consistent and emphasized recommendations to perform lumbar puncture, especially in patients without plausible SE etiology are needed by international guidelines on SE management.

Supplementary Information The online version contains supplementary material available at https://doi.org/10.1007/s00415-021-10447-3.

Author contributions RS and TD planned and designed the study. RS, SMB, and SS acquired and interpreted the data. RS and ST-S analyzed the data and wrote the manuscript. SMB, SS, GMDM, SH, SR, and SM interpreted the data, revised the manuscript, and substantially contributed to the inaugural draft. All authors approved the final submitted version. ST-S and RS conducted statistical analysis.

Funding Open Access funding provided by Universität Basel (Universitätsbibliothek Basel). The study was funded by the University Hospital Basel. The funder (University Hospital Basel) had no role in the design of the study; in the collection, analyses, or interpretation of data; in the writing of the manuscript, or in the decision to publish the results. This study was performed and designed without the input or support of any pharmaceutical company or other commercial interest.

Data availability Anonymized data are available from the corresponding author and will be shared on reasonable request from any qualified investigator.

\section{Compliance with ethical standards}

Conflicts of interest Tolga D Dittrich reports no disclosures. Sira Baumann reports no disclosures. Saskia Semmlack reports no disclosures. Gian Marco De Marchis was or is supported by the Swiss National Science Foundation; Science Funds of the University Hospital Basel and University of Basel; Bangerter-Rhyner-Stiftung; Swisslife Jubiläumsstiftung for Medical Research; Swiss Neurological Society; Fondazione Dr. Ettore Balli; De Quervain research grant; Thermo Fisher GmbH. $\mathrm{He}$ received travel honoraria by Bayer and speaker honoraria by Medtronic and BMS/Pfizer. Sabina Hunziker reports no disclosures. Stephan Rüegg received unconditional research grants from UCBpharma. He received honoraria from serving on the scientific advisory boards of Desitin, Eisai, GSK, and UCB-pharma, travel grants from GSK, Janssen-Cilag, UCB-pharma, speaker fees from UCB-pharma and from serving as a consultant for Eisai, GlaxoSmithKline, Janssen-Cilag, Pfizer, Novartis, and UCB-pharma. He does not hold any stocks of any pharmaceutical industries or manufacturers of medical devices. He received funding from UCB-pharma, Novartis, and Swiss National Science Foundation Grants: grant number 320030_169379/1 and co-applicant for grants numbers 33CM30_125115/1 and 33CM30_140338/1; he disclosed that he is the past-president of the Swiss League against Epilepsy (no payments), Editor of German League against Epilepsy (no payments), and Editor of the Swiss EEG Bulletin (payments from UCB); he received honoraria from serving on the scientific advisory boards of Arvelle, Desitin, Eisai, GlaxoSmithKline (GSK), and UCB-pharma, travel grants from GSK, Janssen-Cilag, UCB-pharma, speaker fees from UCB-pharma and from serving as a consultant for Arvelle, Eisai, GSK, Janssen-Cilag, Pfizer, Novartis, Sandoz, and UCB-pharma. Stephan Marsch reports no disclosures. Sarah Tschudin-Sutter is a member of the Astellas and MSD Advisory Boards for C. difficile, of the Pfizer Antiinfectives Advisory Board for and reports grants from the Swiss National Science Foundation NRP72 (407240_167060), the Bangerter-Rhyner Stiftung, the 
Fonds zur Förderung von Lehre und Forschung der Freiwilligen Akademischen Gesellschaft Basel, and the Jubiläumsstiftung from Swiss Life. Raoul Sutter received research grants from the Swiss National Foundation (No 320030_169379), the Research Fund of the University Basel, the Scientific Society Basel, and the Bangerter-Rhyner Foundation. He received personal grants from UCB-pharma and holds stocks from Novartis, Roche, Alcon, and Johnson \& Johnson.

Ethical approval and consent to participate Prior to the initiation of the study the Swiss ethics committee (Ethikkommission Norwest- und Zentralschweiz) approved the study concept in compliance with the ethical standards laid down in the 1964 Declaration of Helsinki and its later amendments and waived patients' consent.

Open Access This article is licensed under a Creative Commons Attribution 4.0 International License, which permits use, sharing, adaptation, distribution and reproduction in any medium or format, as long as you give appropriate credit to the original author(s) and the source, provide a link to the Creative Commons licence, and indicate if changes were made. The images or other third party material in this article are included in the article's Creative Commons licence, unless indicated otherwise in a credit line to the material. If material is not included in the article's Creative Commons licence and your intended use is not permitted by statutory regulation or exceeds the permitted use, you will need to obtain permission directly from the copyright holder. To view a copy of this licence, visit http://creativecommons.org/licenses/by/4.0/.

\section{References}

1. Agan K, Afsar N, Midi I, Us O, Balosso S, Aykut-Bingol C (2009) Predictors of refractoriness in a Turkish status epilepticus data bank. Epilepsy Behav 14:651-654

2. Beuchat I, Novy J, Rossetti AO (2017) Newer antiepileptic drugs in status epilepticus: prescription trends and outcomes in comparison with traditional agents. CNS Drugs 31:327-334

3. Breiner A, Moher D, Brooks J, Cheng W, Hegen H, Deisenhammer F, McCudden CR, Bourque PR (2019) Adult CSF total protein upper reference limits should be age-partitioned and significantly higher than $0.45 \mathrm{~g} / \mathrm{L}$ : a systematic review. J Neurol 266:616-624

4. Brophy GM, Bell R, Claassen J, Alldredge B, Bleck TP, Glauser T, Laroche SM, Riviello JJ Jr, Shutter L, Sperling MR, Treiman DM, Vespa PM (2012) Guidelines for the evaluation and management of status epilepticus. Neurocrit Care 17:3-23

5. Charlson ME, Pompei P, Ales KL, MacKenzie CR (1987) A new method of classifying prognostic comorbidity in longitudinal studies: development and validation. J Chronic Dis 40:373-383

6. Dittrich T, Marsch S, Egli A, Ruegg S, De Marchis GM, TschudinSutter S, Sutter R (2020) Predictors of infectious meningitis or encephalitis: the yield of cerebrospinal fluid in a cross-sectional study. BMC Infect Dis 20:304

7. Dittrich T, Marsch S, Ruegg S, De Marchis GM, Tschudin-Sutter S, Sutter R (2020) Delirium in meningitis and encephalitis: emergence and prediction in a 6-Year Cohort. J Intensive Care Med epub ahead of print

8. Evison J, Aebi C, Francioli P, Péter O, Bassetti S, Gervaixe A, Zimmerli S, Weber R (2005) Abklärung und Therapie der Lyme-Borreliose bei Erwachsenen und Kindern. Schweizerische Ärztezeitung 86:2375-2384

9. Fatuzzo D, Novy J, Rossetti AO (2018) Use of newer antiepileptic drugs and prognosis in adults with status epilepticus: comparison between 2009 and 2017. Epilepsia 59:e98-e102
10. Gaspard N, Foreman BP, Alvarez V, Cabrera Kang C, Probasco JC, Jongeling AC, Meyers E, Espinera A, Haas KF, Schmitt SE, Gerard EE, Gofton T, Kaplan PW, Lee JW, Legros B, Szaflarski JP, Westover BM, LaRoche SM, Hirsch LJ, Critical Care EEGMRC (2015) New-onset refractory status epilepticus: etiology, clinical features, and outcome. Neurology 85:1604-1613

11. Glauser T, Shinnar S, Gloss D, Alldredge B, Arya R, Bainbridge J, Bare M, Bleck T, Dodson WE, Garrity L, Jagoda A, Lowenstein D, Pellock J, Riviello J, Sloan E, Treiman DM (2016) Evidence-based guideline: treatment of convulsive status epilepticus in children and adults: report of the guideline committee of the American epilepsy society. Epilepsy Curr 16:48-61

12. Graus F, Titulaer MJ, Balu R, Benseler S, Bien CG, Cellucci T, Cortese I, Dale RC, Gelfand JM, Geschwind M, Glaser CA, Honnorat J, Hoftberger R, Iizuka T, Irani SR, Lancaster E, Leypoldt F, Pruss H, Rae-Grant A, Reindl M, Rosenfeld MR, Rostasy K, Saiz A, Venkatesan A, Vincent A, Wandinger KP, Waters P, Dalmau J (2016) A clinical approach to diagnosis of autoimmune encephalitis. Lancet Neurol 15:391-404

13. Hirsch LJ, Gaspard N, van Baalen A, Nabbout R, Demeret S, Loddenkemper T, Navarro V, Specchio N, Lagae L, Rossetti AO, Hocker S, Gofton TE, Abend NS, Gilmore EJ, Hahn C, Khosravani H, Rosenow F, Trinka E (2018) Proposed consensus definitions for new-onset refractory status epilepticus (NORSE), febrile infection-related epilepsy syndrome (FIRES), and related conditions. Epilepsia 59:739-744

14. Knake S, Rosenow F, Vescovi M, Oertel WH, Mueller HH, Wirbatz A, Katsarou N, Hamer HM (2001) Incidence of status epilepticus in adults in Germany: a prospective, population-based study. Epilepsia 42:714-718

15. Meierkord H, Boon P, Engelsen B, Gocke K, Shorvon S, Tinuper P, Holtkamp M, European Federation of Neurological S (2010) EFNS guideline on the management of status epilepticus in adults. Eur J Neurol: Off J Eur Federation Neurol Soc 17:348-355

16. Novy J, Logroscino G, Rossetti AO (2010) Refractory status epilepticus: a prospective observational study. Epilepsia 51:251-256

17. Rossetti AO, Hurwitz S, Logroscino G, Bromfield EB (2006) Prognosis of status epilepticus: role of aetiology, age, and consciousness impairment at presentation. J Neurol Neurosurg Psychiatry 77:611-615

18. Rossetti AO, Logroscino G, Bromfield EB (2006) A clinical score for prognosis of status epilepticus in adults. Neurology 66:1736-1738

19. Rossetti AO, Logroscino G, Milligan TA, Michaelides C, Ruffieux C, Bromfield EB (2008) Status Epilepticus Severity Score (STESS): a tool to orient early treatment strategy. J Neurol 255:1561-1566

20. Solnes LB, Jones KM, Rowe SP, Pattanayak P, Nalluri A, Venkatesan A, Probasco JC, Javadi MS (2017) Diagnostic value of (18)F-FDG PET/CT versus MRI in the setting of antibody-specific autoimmune encephalitis. J Nucl Med 58:1307-1313

21. Strzelczyk A, Ansorge S, Hapfelmeier J, Bonthapally V, Erder MH, Rosenow F (2017) Costs, length of stay, and mortality of super-refractory status epilepticus: a population-based study from Germany. Epilepsia 58:1533-1541

22. Sutter R, Kaplan PW, Rüegg S (2013) Independent external validation of the Status Epilepticus Severity Score. Crit Care Med 41:e475-479

23. Sutter R, Kaplan PW, Rüegg S (2013) Outcome predictors for status epilepticus-what really counts. Nat Rev Neurol 9:525-534

24. Sutter R, Marsch S, Fuhr P, Kaplan PW, Rüegg S (2014) Anesthetic drugs in status epilepticus-risk or rescue? A six-year cohort study. Neurology 82:656-664

25. Sutter R, Marsch S, Fuhr P, Rüegg S (2013) Mortality and recovery from refractory status epilepticus in the ICU: a 7-year observational study. Epilepsia 54:502-511 
26. Sutter R, Rüegg S (2013) Predicting outcome in adults with status epilepticus. Zeitschrift für Epileptologie 26:79-84

27. Sutter R, Semmlack S, Kaplan PW (2016) Nonconvulsive status epilepticus in adults-insights into the invisible. Nat Rev Neurol 12:281-293

28. Sutter R, Tschudin-Sutter S, Grize L, Fuhr P, Bonten MJ, Widmer AF, Marsch S, Ruegg S (2012) Associations between infections and clinical outcome parameters in status epilepticus: a retrospective 5-year cohort study. Epilepsia 53:1489-1497

29. Trinka E, Cock H, Hesdorffer D, Rossetti AO, Scheffer IE, Shinnar S, Shorvon S, Lowenstein DH (2015) A definition and classification of status epilepticus-Report of the ILAE Task Force on Classification of Status Epilepticus. Epilepsia 56:1515-1523

30. Venkatesan A, Tunkel AR, Bloch KC, Lauring AS, Sejvar J, Bitnun A, Stahl JP, Mailles A, Drebot M, Rupprecht CE, Yoder J, Cope JR, Wilson MR, Whitley RJ, Sullivan J, Granerod J, Jones C, Eastwood K, Ward KN, Durrheim DN, Solbrig MV, Guo-Dong L, Glaser CA, International Encephalitis C (2013) Case definitions, diagnostic algorithms, and priorities in encephalitis: consensus statement of the international encephalitis consortium. Clin Infect Dis 57:1114-1128
31. von Arxa S, Leibb SL, Sturzenegger M, Sendi P (2017) Infektionen des zentralen Nervensystems-Teil 2: Enzephalitis beim Erwachsenen. Schweiz Med Forum 17:492-498

32. von Elm E, Altman DG, Egger M, Pocock SJ, Gotzsche PC, Vandenbroucke JP, Initiative S (2007) The strengthening the reporting of observational studies in epidemiology (STROBE) statement: guidelines for reporting observational studies. Lancet 370:1453-1457

33. Yamamoto W, Ogusa E, Matsumoto K, Maruta A, Ishigatsubo Y, Kanamori H (2014) Human herpesvirus-6 encephalopathy after hematopoietic stem cell transplantation and class I human leukocyte antigen. Clin Transplant 28:540-545

34. Yanagida A, Kanazawa N, Kaneko J, Kaneko A, Iwase R, Suga H, Nonoda Y, Onozawa Y, Kitamura E, Nishiyama K, Iizuka T (2020) Clinically based score predicting cryptogenic NORSE at the early stage of status epilepticus. Neurol Neuroimmunol Neuroinflamm 7(5)

35. Zelano J, Moller F, Dobesberger J, Trinka E, Kumlien E (2014) Infections in status epilepticus: a retrospective 5-year cohort study. Seizure 23:603-606 